\title{
Disabling hip osteoarthritis: gender, body mass, health and functional status correlates
}

\author{
Ray Marks \\ School of Health \& Behavioral Sciences, City University of New York, York College, and Department of Health \& Behavior Studies, \\ Columbia University, Teachers College, New York, USA; rm226@columbia.edu
}

Received 12 February 2010; revised 15 March 2010; accepted 16 March 2010.

\begin{abstract}
Objective: To examine gender differences in self-reported pain and function before and after hip replacement surgery and the extent to which overweight, comorbidities and muscular status impact pain and function in adults with disabling end-stage hip joint osteoarthritis. Setting: Orthopedic Hospital Setting on the East Coast of the United States. Study Design: Cross-sectional retrospective chart review. Methods: The desired demographic, physical and psychological attributes of 1040 adults with end-stage hip osteoarthritis hospitalized for hip surgery were recorded and subjected to comparison and correlational analyses. These data included gender, self-reported weight, height, numbers and nature of physical and psychological comorbidities, pain intensity, ambulatory capacity and discharge destination. Sub-group analyses of 808 candidates hospitalized for primary unilateral surgery were also conducted using SPSS 16. Results: There were significant $(p<0.05)$ associations between gender, pain scores, comorbidity numbers and ambulatory capacity. Specifically, women who exhibited higher comorbid disease rates than men, exhibited higher pre-surgery pain levels and greater functional limitations in walking ability before and after surgery than men with the same condition. In sub-group analyses of men and women with the same mean age, comorbid prevalence rates, and body mass indices, women were found to have significantly higher ideal weights on average than men, and those with higher ideal weights recovered more slowly after surgery ( $p$ $<0.05$ ). Conclusion: The presentation of hip joint osteoarthritis is not uniform, and may be impacted differentially by gender. Women with high ideal body weights, may be specifically
\end{abstract}

impacted. Whether genetic or other factors account for gender differences in pain and function among adults with disabling hip osteoarthritis observation needs to be examined.

Keywords: Body Mass; Comorbidity; Function; Gender; Hip Joint; Osteoarthritis

\section{INTRODUCTION}

Hip joint osteoarthritis, a prevalent medical condition, causes considerable distress and chronic disability among community-dwelling adults aged 55 years and older in all countries. Associated with substantial direct as well as indirect costs, a number of factors other than aging may influence its impact. These factors include the presence of one or more comorbid health conditions [1] varying degrees of pain [2], variations in the disease itself [3], gender [4], high body weights [5], as well as proprioceptive, muscle strength and hip flexion range of motion deficits. In addition, prior musculoskeletal injuries, excessive occupational stresses and systemic factors may impact the severity of osteoarthritis [5].

Because hip osteoarthritis, a leading cause of functional disability [4] is related to poor-self related health [6], and causes great suffering, but is often approached quite uniformally as far as treatment is concerned [7], it was felt a better understanding of the disease and its clinical heterogeneity [8] might prove beneficial. In particular, it was felt that if further evidence for important subsets in the expression of the disease could be identified, more targeted efforts to reduce the significant social and economic burden of the disease might be forthcoming in the future. Factors such as prevailing mental and physical impairments, other than hip osteoarthritis and their interaction with pain and functional capacity have also received limited attention in the related literature [3].

To bridge this gap, this work examined the association 
between gender, pain and function and potentially preventable or treatable comorbid health conditions, obesity, and lower leg weakness as experienced by a hospitalized cohort of community dwelling adults with end-stage hip osteoarthritis. More specifically, this analysis attempted to discern if there are any clinically relevant gender differences in the pain experience and functional status of individuals of similar sociodemographic backgrounds and disease severity, and whether this was impacted by the presence of prevailing age, body mass, comorbid health conditions, and other factors such as muscle strength capacity.

The primary research questions driving this investigation were the following:

1) Is the extent of the disability experienced by adults with disabling hip joint osteoarthritis similar for men and women?

2) What coexisting health conditions are most commonly observed among community dwelling adults with end stage hip joint osteoarthritis and is this uniform for men and women?

3) Is the level of pain and mobility experienced by adults with disabling hip joint osteoarthritis influenced by body mass and/or the presence of one or more preexisting medical conditions?

4) Is the level of pain and reduced mobility experienced by adults with disabling hip joint osteoarthritis influenced by the presence of muscle weakness as estimated on a numeric scale?

\section{MATERIALS AND METHODS}

All available medical records of community-dwelling adults hospitalized for hip replacement due to clinically and radiographically diagnosed end-stage hip osteoarthritis of one or both hips were examined. These data had been collected prospectively over a 10 month period as part of an approved parent study of hip joint surgical outcomes, but with no active patient involvement. Patients with acute hip fractures or any other primary diagnosis were excluded.

\subsection{Study Sample}

The cohort examined included 1040 men and women between the ages of 23-89 years diagnosed as having definitive clinical and radiographic evidence of osteoarthritis of one or both hip joints requiring primary or secondary surgery as determined by an orthopedic surgeon. The majority came from the local community.

\subsection{Procedures}

The desired data were systematically extracted by the researcher from the patient's medical record. These data, which had been systematically recorded by the attending physician[s], nurse[s] and physical therapist, included age, gender, self-reported height and weight. They also included the primary reason for hospital admission, the presence and nature of any pre-existing orthopedic problems, comorbid physical and/or mental health conditions other than hip disease, and symptomatic selfreported baseline pain estimates on a 5-point ordinal scale where 1 was minimal pain and 5 was maximal pain. Muscle weakness of the affected limb as identified by a composite of physical tests including manual muscle tests of the knee and hip muscles rated from 5-1 (no problem-severe loss of strength) and active hip flexion joint range of motion estimates of the affected hip (in degrees), plus patient perceived pre-operative walking status and distance in blocks and 3-day post-operative walking distance as recorded in meters by the physical therapist were also recorded. Separate calculations on the chart included those of body mass index (weight/ height ${ }^{2}$ ) and ideal weight estimates (calculated for each individual based on gender, age, height, and weight from standard estimates that represent average medically recommended values) and expressed as a percentage of the patient's actual body weight. Also recorded was the disease duration, as well as presence of any other affected joints and prior surgeries. The data were entered systematically onto an Excel spreadsheet and transposed thereafter, into SPSS version 16.0 files to describe the sample and to analyze the data with regard to age (above and below 60 years of age), gender, comorbid health numbers, and pain and functional variables among the observed hip osteoarthritis surgical candidates using chi-square tests, cross-tabulations, and analysis of variance, as indicated. An a priori significance level of 0.05 was adopted.

\section{RESULTS}

\subsection{General features}

The 1040 cases presently studied had a mean age of $65.36 \pm 13.04$ years, with a median age of 68 years, and a mode of 71 years. As shown in Table 1, approximately $30 \%$ or 312 were 60 years or under in age. The number hospitalized for primary unilateral surgery was 808 or approximately $78 \%$, 53 or 5\% required bilateral surgery, and 179 or $17.2 \%$ were undergoing revision surgery. Overall, the present sample was constituted by more females than males (60\% vs $40 \%$ ), but among cases under the age of 60 , the numbers of hospitalized men and women were comparable (158 vs 163). Among this cohort, there were 9 reported comorbid health domains related to different body systems as outlined by Dyke et 
al. [1], excluding obesity, including those shown in Table 2 . The frequency of patients having reported either a mental or physical pre-existing health condition ranged from 0 or no co-existing problem to 5 , with a mean of 1 \pm 1 , and a median of $1 ; 36 \%$ had no comorbid conditions, $30.5 \%$ had 1 condition, $22.9 \%$ had 2 conditions, 6.7\% had 3 conditions, $1.2 \%$ had 4 health conditions, and $0.2 \%$ had 5 co-existing conditions. The majority or $69.5 \%$ of cases had at least one additional pre-existing physical health condition and the numbers of reported comorbid health conditions increased with age $(r=0.232$; $\mathrm{p}=0.001)$. That is, those who were 60 years and older had more evidence of the presence of one or more chronic health conditions than those below 60 years of age.

When analyzed separately with regard to gender, on average, women tended to slightly more comorbid conditions than men (CIs being 1.06-1.22; 0.83-1.00, respectively), although the median of 1 , and range $0-5$ were comparable $\left(\mathrm{X}^{2}=18.61 ; d f=15 ; \mathrm{p}=0.232\right)$. In addition to the high prevalence of having at least one co-existing physical problem, $52.7 \%$ of the cohort studied had a pre-existing orthopaedic problem such as a fracture, or congenital bone problem, and in contrast to medical comorbidity prevalence, men had higher rates of co-existing orthopaedic related problems than women $\left(\mathrm{X}^{2}=9.98 ; d f=9 ; \mathrm{p}=0.001\right)$. Women had higher rates of autoimmune diagnoses related to arthritis $\left(X^{2}=9.42\right.$; $d f=9 ; \mathrm{p}=0.001$ ), and cardiac disease including hypertension, heart murmurs and defective valve conditions were observed most commonly among the diseases listed on the charts.

In comparing those requiring bilateral hip surgery with those hospitalized for primary unilateral surgeries, these patients were found to be younger on average with a mean age of $54.11 \pm 13.18$ years versus a mean age of $66.03 \pm 12.4$ years. Their comorbidity level was also

Table 1. Summary of key baseline demographic and disease related characteristics of the hip osteoarthritis cohort. Values are means and standard deviations and percentages of total sample.

\begin{tabular}{|c|c|c|c|}
\hline Characteristics & Overall Group N = 1037 & Women $\mathbf{N}=\mathbf{6 0 0}$ & Men N = 437 \\
\hline \multicolumn{4}{|l|}{ Demographics } \\
\hline Age (years) & $65.36 \pm 13$ & $67.22 \pm 12.96$ & $62.74 \pm 12.70^{*}$ \\
\hline \# under 60 yrs & $316(30.4 \%)$ & 153 & 163 \\
\hline \# over 60 yrs & 723 & 447 & $274 *$ \\
\hline Body mass index $\left(\mathrm{kg} \cdot \mathrm{m}^{-2}\right)$ & $27.5 \pm 5.7$ & $26.6 \pm 5.9$ & $28.7 \pm 5.1^{*}$ \\
\hline Morbidity Count & $1.0 \pm 1.0$ & $1.2 \pm 0.04$ & $0.92 \pm 0.04 *$ \\
\hline \multicolumn{4}{|l|}{ (Range 0-5) } \\
\hline 0 & $37 \%$ & $19 \%$ & $17.5 \%$ \\
\hline 1 & $31.2 \%$ & $18.9 \%$ & $12.3 \%$ \\
\hline 2 & $23.4 \%$ & $13.5 \%$ & $9.9 \%$ \\
\hline 3 & $6.8 \%$ & $5.1 \%$ & $1.7 \%$ \\
\hline 4 & $1.3 \%$ & $1.2 \%$ & $0.2 \%$ \\
\hline 5 & $0.2 \%$ & $0.1 \%$ & $0.2 \%$ \\
\hline Concomitant orthopedic problem & $52 \%$ & $25.2 \%$ & $34 \% *$ \\
\hline Autoimmune related diagnosis & $5.6 \%$ & $4.5 \%$ & $2.2 \% *$ \\
\hline Depression history & $6.4 \%$ & $5 \%$ & $1.4 \% *$ \\
\hline
\end{tabular}

*men were significantly younger than women admitted for the same surgery $\mathrm{p}=0.001$

*fewer men over 60 yrs were admitted than women in present cohort $\mathrm{p}=0.001$

*men had fewer reported comorbid conditions ( $\mathrm{p}=0.002)$

*men had higher body mass indices in general than the women, as well as more orthopedic problems than women $\mathrm{p}=0.001$

*men had fewer autoimmune and depression history related diagnoses than women $\mathrm{p}=0.001$ 
Table 2. Summary of extent of comorbid diseases noted on charts at baseline among hip osteoarthritis surgical cases when disaggregated by gender showing significant gender differences $(N=560)$.

\begin{tabular}{lcc}
\hline \multicolumn{3}{c}{ GENDER } \\
\hline \multicolumn{1}{c}{ Comorbid condition } & Female N (\%) & Male N (\%) \\
\hline Cardiovascular disease & $187(33 \%)$ & $124(22 \%)$ \\
Hypothyroidism & $47(8.3 \%)$ & $5(0.76 \%)$ \\
Osteoporosis & $46(8.8 \%)$ & $1(1.8)$ \\
Cancer & $36(6.4 \%)$ & $22(3.9 \%)$ \\
Depression & $28(5 \%)$ & $8(1.3 \%)$ \\
Autoimmune disease & $28.5(5 \%)$ & $13(2.3 \%)$ \\
Diabetes & $20(3.5 \%)$ & $13(2.3 \%)$ \\
Asthma & $16(3.9 \%)$ & $20(2.8 \%)$ \\
Stroke & $5(0.75 \%)$ & 0 \\
Kidney disease & $4(0.71 \%)$ & 0 \\
Prior injury/bone defect & $98(17.5 \%)$ & $91(16.2 \%)$ \\
\hline
\end{tabular}

Note: Some cases have more than one comorbid disease.

No records of visual or hearing impairments were available.

Cardiovascular disease category includes high blood pressure, or any pre-existing heart condition or vascular disease.

lower than those undergoing unilateral surgery ( $\mathrm{p}=$ 0.001), and was independent of age, when assessed using cross tabulations. It was thus deemed of interest to specifically examine only those undergoing unilateral surgery, as it was felt, those with bilateral hip joint osteoarthritis may have had a different health profile, in general from the majority of the primary surgical cases. Those hospitalized for a previous procedure, revision or re-operation were also excluded.

\subsection{Related Subgroup Analyses}

In a further analysis of the 808 cases undergoing primary unilateral surgery, median age, 68 years, it was observed that this sub-group had one comorbid health problem on average and the majority of these cases or $69.2 \%$ were overweight or obese with a mean body mass index of $27.6 \pm 5.5$. Among those unilateral cases with comorbid health conditions, the most common condition observed was hypertension alone, or in combination with coronary artery or cardiac disease. Next, injuries, bone disease or prior surgeries were reported most often. The other conditions were hypothyroidism, osteoporosis, and cancer. Women had higher rates of all conditions except asthma and stroke, and lower rates of prior injury than men.

To better control for potential confounders, a further sub-analysis of 100 age-matched cases, mean age $66.3 \pm$ 10.3 years (66.52 \pm 10.98 years of age for the 46 women; $66.06 \pm 9.69$ years of age for the 54 men, $p=0.822$ ), with comparable body mass indices of 27.7 and 27.9, for women and men, respectively ( $p=0.432$ ), and an average of 1.2 comorbid conditions was undertaken as shown in Table 3. Again, this analysis revealed the subjective pain levels experienced before surgery were found to be significantly higher among the women compared to the men $(3.2 \pm 0.84$ versus $2.7 \pm 0.9$ on a 5 point Likert scale, respectively) ( $p=0.004)$, even though numbers of affected joints were comparable $(p=0.125)$. As well, men in the cohort tended to be able to walk further on average than women before surgery, as well as three days after surgery ( $p=0.011 ; p=0.018$, respectively). While these differences could not be explained on the basis of comorbity numbers or body mass index (w.h ${ }^{-2}$ ) because comorbidity rates were comparable, and a similar percentage were overweight or obese (48\% men vs $50 \%$ women), the ideal body weights estimates calculated for women were 134 percent (CI 113-155) and 106 percent (CI 89-123) for men $(p=0.002)$ with a mean of $124.8+25.3$ for the group. There was also a significant inverse relationship between the ideal weight estimates and the ability to walk one day after surgery $(r=-0.246$, $\mathrm{p}=0.043$ ), but post surgical walking distance was not affected by age, pain, comorbid status or body mass. On the whole, weakness of the lower leg was evident in $40 \%$ cases, and of these cases, $7 \%$ had weakness of both the hip and knee muscles. Although walking endurance at baseline was generally worse if the knee extensors and or the hip and knee extensors on the operative side were found to be weak, this was not significant ( $p=0.196)$. More subjects however, used devices to help them walk if they reported weakness of the lower leg $(p=0.009)$, and a higher percentage of women exhibited a lower leg strength loss than men (61\% vs 34\%). Although the cohort were clearly challenged physically speaking, and disease histories extended from 1 year to 18 years, pain levels were not influenced by disease duration and only $5 \%$ women and 1.3\% men had depression histories. Walking distance and stair walking was affected by numbers of affected joints, but was independent of muscle strength. Device use was correlated positively with age.

\section{DISCUSSION}

Hip joint osteoarthritis, a debilitating condition that increases in prevalence with age, presents an enormous challenge to the health care system worldwide due to its chronicity. To improve the outcomes for this group of 
Table 3. Table showing relationships between gender and walking capacity before and after surgery when controlling for pain in a sub-group of cases older than 60 years of age $(\mathrm{N}=100)$.

\begin{tabular}{ccccccc}
\hline Gender & Age Yr & Walk Dist Blocks & Comorbid Number & Day 3 walk dist Feet & BMI w.h ${ }^{-2}$ & Ideal wt Percent \\
\hline F N = 46 & $66.5 \pm 10.9$ & $2.71 \pm 2.89$ & $1.11 \pm 0.89$ & $4.95 \pm 43.1$ & $527.2 \pm 5.2$ & $132.8 \pm 25.3$ \\
M N = 54 & $66.1 \pm 9.7$ & $4.61 \pm 3.9^{* *}$ & $1.24 \pm 0.8$ & $88.78 \pm 43^{*}$ & $27.9 \pm 4.2$ & $118.6 \pm 18.7^{* *}$ \\
\hline
\end{tabular}

** correlation is significant at 0.01 level (2-tailed)

* correlation is significant at the 0.05 level (2-tailed)

adults, careful evaluation of the underlying pathophysiological and contributing factors to the disease has become essential. To this end, this present retrospective analysis examined trends and interrelationships that might exist among selected physical and health related correlates of the condition and patient characteristics believed to impact on osteoarthritis disability. In particular, a better understanding of mediating, moderating, or causative factors such as gender, body mass, comorbid health status, and muscle weakness and the clinical parameters of pain and functional disability was sought. The goal was to identify new perspectives or to further support current perspectives about what may be most appropriate for purposes of reducing the disability and handicap of hip joint osteoarthritis.

To this end, patients with symptomatic end-stage hip osteoarthritis, and well defined radiological progression were selected for study as there has been no universal agreement as to what stage of progression might be highly important to examine in the context of the natural history of the disease, and which has been poorly evaluated in the past [8]. All had well defined clinical as well as functional disability that had progressed towards its end stage, and the present study examined the variation in disease presentation as regards health status, gender, functional ability and pain among other factors to improve our understanding of this condition. Patient subsets were identified and examined to ascertain if any would be more affected clinically by the disease than others, and if so, if these displayed any unique physical and health status characteristics.

While the current retrospective chart review approach can be seen as limitation, this exploratory study tried to include the entire population of patients with active disease who were admitted for primary or seconddary hip surgery over a 10 month period and examined their short-term functional recovery rates post-surgery. Although it is recognized that the present findings could be biased by who was studied, the age range of the studied cohort was quite typical of those described in the literature $[7,9]$ and consistent with several other studies $[7,10]$, a high percentage were women. As well, a high majority of the cases presently reviewed reported having at least one co-existing comorbid physical health condition, a finding identified by Van Dijk et al. [2] for adults with osteoarthritis of the hip and knee. Moreover, consistent with findings by Tuminen et al. [9], those older than 60 years of age had significantly higher comorbidity rates than those under 60 years of age (See Table 4).

In addition, although contrary to findings of Franklin et al. [11] in an Icelandic case control study, a consistently high percentage of the study cohort were overweight or obese as was observed by Dijk et al. [2]. While obesity can be regarded as a comorbid health condition in its own right $[2,8]$, this health indicator was presently analyzed separately from the other reported comorbid health conditions, along with estimates of the subject's ideal body weight estimates expressed as a percentage. In this respect, more than two thirds of the men and women presently studied were overweight or obese and women had higher percentages of ideal weight estimates than men when controlling for age, body mass, and comorbidity number, suggesting women with this disease are more overweight in general than men, when considering their height and age. In addition, there were gender differences in pre-surgical pain levels and functional mobility at baseline and post-surgery, in favour of the men, and different distributions of comorbid health conditions between men and women.

The higher than desirable numbers of comorbid health conditions, autoimmune conditions, and high body weights especially among women, are important to note, because, as has been observed in other studies, hip osteoarthritis is more frequently part of a polyarticular disease, with greater symptomatic and structural severity [7]. In addition, comorbidity and high body mass are risk factors for functional activity limitations and pain $[2,3,9]$ and greater deterioration of hip joint disease [12]. Obesity is also correlated with higher mortality rates [11] and poorer health status. While the severity of the comorbidity was not studied, consistent with findings by Van Dijk et al. [2] the most common coexisting disease presently observed was heart disease or hypertension, a health condition expected to influence both activity and pain levels, as well as the outcomes of options for surgery and rehabilitation. 
Table 4. Sample of past studies that support some of the present findings and the hypothetical disability model.

\begin{tabular}{|c|c|c|}
\hline Authors & Sample & Key Findings \\
\hline \multirow[t]{2}{*}{ Jorring [13] } & $\begin{array}{l}6321 \text { cases of which } \\
4.7 \% \text { had hip OA }\end{array}$ & In all age groups above 60 hip OA was twice as common in women as in men \\
\hline & & The condition was more severe radiologically in women who more handicapped than men \\
\hline Juahkoski et al. [14] & 118 cases of hip OA & Comorbidity (CM) number influenced pain/function \\
\hline Juhakoski et al. [15] & 840 cases hip OA & Heavy manual labor + injury were predictive for hip OA \\
\hline \multirow[t]{3}{*}{ Kadam et al. [16] } & 11375 OA cases & CM for OA was extensive compared to controls \\
\hline & & Non musculoskeletal conditions observed included obesity, heart disease, phlebitis \\
\hline & & CM was not explained by age, gender or social class \\
\hline Katz et al. [17] & Patients undergoing hip & Women had worse functional status than men surgery for hip OA \\
\hline Maiilefert et al. [7] & 508 patients with hip OA & $\begin{array}{l}\text { Hip OA in women is more frequently part of a polyarticular OA and displays greater symptomatic } \\
\text { and structural severity }\end{array}$ \\
\hline \multirow[t]{3}{*}{ Roseman et al. [18] } & 1250 OA patients & Main predictor of disability included body mass \\
\hline & & Impact of OA differed between genders \\
\hline & & More women than men constituted the sample \\
\hline Tepper et al. [19] & 73 cases of hip OA & $\begin{array}{l}\text { Age/hip trauma were associated with hip OA in men } \\
\text { Obesity was associated with bilateral hip OA }\end{array}$ \\
\hline Tuominen et al. [9] & 893 patients & $\begin{array}{l}649 \text { or } 73 \% \text { had CM, mean no. = } 2 \\
\text {-At baseline health-related QOL was lower in those with CM }\end{array}$ \\
\hline \multirow[t]{3}{*}{ Van Dijk et al. [2] } & 288 elderly-hip/knee OA & Almost all had at least comorbidity \\
\hline & & CM was related to activity limitations/pain \\
\hline & & $\begin{array}{l}\text { Most common CM condition = cardiac diseases } \\
\text { Overweight and obesity was common }\end{array}$ \\
\hline
\end{tabular}

Note: $\mathrm{CM}=$ comorbidity; $\mathrm{OA}=$ osteoarthritis; $\mathrm{QOL}=$ quality of life

In terms of gender, and as has been observed in other studies, although men and women had an equal chance of being included in the study, women clearly constituted the majority of those hospitalized over the study period. Moreover, even though the prevalence of the disease is probably similar among men and women [7], consistent with findings of other studies [7] women of comparable ages to men with similar health histories, could not walk as far on average with the same condition either before or after surgery. These observations support the view that being female may be an independent risk factor for disabling hip osteoarthritis as outlined by O'Connor [4] and the higher rates of endocrine/etabolic diseases, as well as cardiac diseases among the women may explain their performance-based activity limitations [1]. They may also suffer from higher rates of muscle mass and strength losses due to one or more of these health conditions, as well as high levels of fat infiltration that might impact their ability to function physically as they age [20]. Indeed, although the women and men currently examined had comparable body mass indices, women as a whole exhibited higher ideal body weight estimates as well as higher rates of strength losses of the affected lower leg that can limit performance [1]. They also had higher rates of reported depression and pain.

Men, on the other hand, were more likely to simply be overweight, to have lower rates of comorbid disease and-to have suffered a prior orthopaedic problem, suggesting a somewhat different disease profile. They may thus suffer less or have less widespread intercurrent diseases and related impairments than women and are thus more active functionally before surgery and able to recover more rapidly after surgery, and in the present case were generally younger than the women.

Alternately, the greater disability presently experienced by women than men with the same diagnosis, which has also been observed among knee arthroplasty candidates [21], may indicate women who are candidates 
for hip joint replacement surgery should be preferentially targeted before and after surgery to offset unwarranted functional declines, and the possibility of developing further radiological damage, cardiac diseases and others. The data also show considerable inter-individual differences in disease presentation and suggest more attention to classifying patients according to the presence or absence of comorbidities and mechanical or biological factors may influence the disease outcome as well as body weight, pain and functional capacity quite favorably [14,20-24].

Moreover, because untreated or poorly treated hip osteoarthritis may increase the risk for obesity, as well as related medical conditions, more carefully designed preventive and therapeutic strategies that focus on muscle strengthening are clearly indicated. In addition, to reduce the rate of progression of the disease, and to help the patient to function optimally, those with unrelenting pain might be targeted for treatment early on [6,25]. The higher rate of thyroid disorders, osteoporosis, and autoimmune conditions experienced by the women in the present analysis suggests additional targets for early intervention as well to offset possible excessive tissue damage and injury. As well, generic efforts to prevent injuries such as falls, may be influential in minimizing the hip osteoarthritis burden, as may more targeted efforts to reduce occupational stresses at work thought to lead to hip osteoarthritis $[5,15]$.

However, it is recognized that the present cohort may not represent all cases with condition; not all data were available or complete; and chart reviews may not be without limitations. Also, even though several comorbid health problems and their frequency of occurrence were recorded, their severity was not. Visual impairments as well as hearing impairments were also not recorded for the hip osteoarthritis cases were studied. These conditions, and their prevalence and relationship to hip osteoarthritis disability thus need to be studied further to assess if concerted efforts to prevent and treat comorbid conditions, as well as depression and pain may lessen the disability associated with hip joint osteoarthritis and its negative impact on functional independence and life quality.

In summary, factors placing adults at risk for hip osteoarthritis disability are age, being female [10], having high body weights [11], and one or more comorbid health conditions. Muscle strength loss, trauma, excessive loading of the hip joint as well as congenital hip problems may also place individuals at risk for hip osteoarthritis as well high rates of further disability. Because over a third of the present cases were under 60 years of age, and had not necessarily experienced trauma, but were heavier on average than those over age 60 years, early and carefully tailored interventions to maximize weight control seems highly desirable. Moreover, given that being obese increases the risk for hip osteoarthritis 2.47 fold among women [26], women might be preferentially targeted. In addition, professionals interested in improving the outlook for adults with hip joint osteoarthritis must acknowledge the broad spectrum of other health problems adults with hip osteoarthritis may face that can impact the need for hip joint replacement [23] as well as postoperative recovery processes [12,27] and the true burden of the disease [28]. In light of its im-

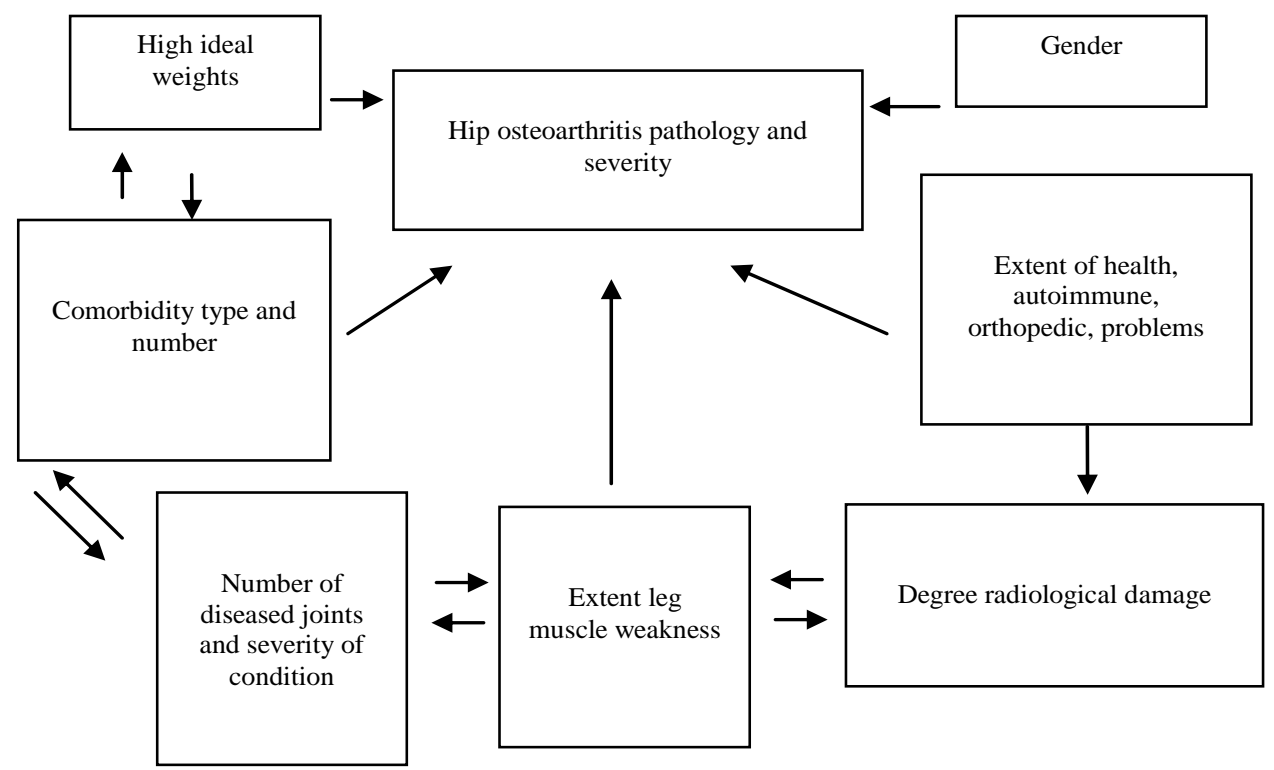

Figure 1. Hypothetical interaction of key variables other than age that might influence outcome of hip osteoarthritis. 
mense variability, and disproportionate impact on women, a comprehensive rather than a uniform clinical pathway for intervening in the hip osteoarthritis degenerative process as advocated by Wang et al. [24] is indicated. In particular, in addition to targeted efforts to assist with weight maintenance or reduction and pain and depresssion control, the efficacy of implant and rehabilitation tailoring should be explored. Assessing comorbidities and the extent and severity of these at baseline may also help to prioritize medical-decision making $[9,26]$ and to thereby tailor care approaches that can potentially reduce hospital stays, as well as the human and economic impact $[13,16-19,25,29]$.

\section{REFERENCES}

[1] Van Dijk, G.M., Veenhof, C., Lankhorst, G.J. and Dekker, J. (2009) Limitations in patients with osteoarthritis of the hip and knee: The relationship with body functions, comorbidity and cognitive function. Disability and Rehabilitation, 31(20), 1685-1691.

[2] Van Dijk, G.M., Veenhof, C., Schellevis, F., Hulsmans, H., Bakker, J.P.J, Arwert, H., Dekker, J.H.M., Lankhorst, G.J. and Dekker, J (2008) Comorbidity, limitations in activities and pain in patients with osteoarthritis. BMC Musculoskeletal Disorders, 9(26), 95.

[3] Joost, D., Van Dijk, G.M. and Veenhof, C. (2009) Risk factors for functional decline in osteoarthritis of the hip or knee. Current Opinion in Rheumatology, 21(5), 520524.

[4] O’Connor, M.I. (2006) Osteoarthritis of the hip and knee: Sex and gender differences. Orthopedic Clinicis of North America, 37(4), 559-568.

[5] Felson, D.T. (2004) An update on the pathogenesis and epidemiology of osteoarthritis. Radiological Clinics of North America, 42(1), 1-9.

[6] Cecchi, F., Mannoni, A., Molino-Lova, R., Ceppatelli, S., Benvenuti, E., Bandinelli, S., Lauretani, F., Macchi, C. and Ferrucci, L. (2008) Epidemiology of hip and knee pain in a community based sample of Italian persons aged 65 and older. Osteoarthritis Cartilage, 16(9), 10391046.

[7] Maillefert, J.F., Guegen, A., Monreal, M., Nguyen, M., Berdah, L., Lequesne M., Mazieres B, Vignon, E. and Dougados, M. (2003) Sex differences in hip osteoarthritis: Results of a longitudinal study in 508 patients. Annals of the Rheumatic Diseases, 62(10), 931-934.

[8] Dougados, M., Guegen, A., Mguyen, M., Berdah, L., Lequesne, M., Mazieres, B. and Vignon, E. (1996) Radiological progression of hip osteoarthritis: Definition, risk factors and correlations with clinical status. Annals of the Rheumatic Diseases, 55(6), 356-362.

[9] Tuominen, U., Blom, M., Hirvonen, J., Seitsalo, S., Lehto, M., Paavolainen, P., Hietaniena, K., Rissanen, P. and Sintonen, H. (2007) The effect of co-morbidities on health-related quality of life in patients placed on the waiting list for total joint replacement. Health and Quality of Life Outcomes, 5(19), 16.
[10] Chung, C.Y., Park, M.S., Lee, K.M., Lee, S.H., Kim, T.K., Kim, K.W., Park, J.H. and Lee, J.J. (2009) Hip osteoarthritis and risk factors in elderly Korean population. Osteoarthritis Cartilage, 18(3), 312-316.

[11] Franklin, J., Ingvarsson, T., Englund, M. and Lohmander, L.S. (2009) Sex differences in the association between body mass index and total hip or knee joint replacement resulting from osteoarthritis. Annals of the Rheumatic Diseases, 68(4), 536-540.

[12] Peters, T.J., Sanders, C., Dieppe. P. and Donovan, J. (2005) Factors associated with change in pain and disability over time: A community-based prospective observational study of hip and knee osteoarthritis. British Journal of General Practice, 55(512), 205-211.

[13] Jorring, K. (1980) Osteoarthritis of the hip. Epidemiology and clinical role. Acta Orthopedica Scandinavica, 51(3), 523-530.

[14] Juhakoski, R., Tenhonen, S., Anttonene, T., Kauppinen, T. and Aroski, J.P. (2008) Factors affecting self-reported pain and physical function in patients with hip osteoarthritis. Archives of Physical Medicine and Rehabilitation, 89(6), 1066-1073.

[15] Juhakoski, R., Heliovaara, M., Impivaara, O., Kroger, H., Knekt, P., Lauren, H. and Aroski, J.P. (2009) Risk factors for the development of hip osteoarthritis: A populationbased prospective survey. Rheumatology (Oxford), 48(1), 83-87.

[16] Kadam, U.T., Jordan, K. and Croft, P.R. (2004) Clinical comorbidity in patients with osteoarthritis: A case-control study of general practice consulters in England and Wales. Annals of the Rheumatic Diseases, 63(4), 408414.

[17] Katz, J.N., Wright, E.A., Guadagnoli, E., Liang, M.H., Karlson, E.W. and Cleary, P.D. (1994) Differences between men and women undergoing orthopedic surgery for degenerative arthritis. Arthritis and Rheumatism, 37(5), 687-694.

[18] Rosemann, T., Laux, G. and Szecsenyi, J. (2007) Osteoarthritis: Quality of life, comorbidities, medication and health service utilization assessed in a large sample of primary care patients. Journal of Orthopedic Surgery Research, 2, 12.

[19] Tepper, S. and Hochberg, M.C. (1993) Factors associated with hip osteoarthritis: Data from the First National Health and Nutrition Examination Survey (NHANES-1) American Journal of Epidemiology, 137(10), 1081-1088.

[20] Rasch, A., Bystrom, A.H., Dalen, N. and Berg, H.E. (2007) Reduced muscle radiological density, cross-sectional area, and strength of major hip and knee muscles in 22 patients with hip osteoarthritis. Acta Orthopaedica, 78(4), 505-510.

[21] Petterson, S.C., Raisis, L., Bodenstab, A. and SnyderMackler, L. (2007) Disease-specific gender differences among total knee arthroplasty candidates. Journal ofBone and Joint Surgery, 89(11), 2327-2333.

[22] Harrison, P.J. and Tunbridge, E.M. (2008) CatecholO-Methyltransferase (COMT): A gene contributing to sex differences in brain function, and to sexual dimorphism in the predisposition to psychiatric disorders. Neuropsychopharmacology, 33(13), 3037-3045.

[23] Dieppe, P., Judge, A., Williams, S., Ikwueke, I., Guenther, K.P., Floeren, M., et al. (2009) Variations in the pre-op- 
erative status of patients coming to primary hip replacement for osteoarthritis in European orthopaedic centers. BMC Musculoskeletal Disorders, 10(1), 19.

[24] Wang, A., Hall, S., Gilbey, H. and Ackland, T. (1997) Patient variability and the design of clinical pathways after primary total hip replacement surgery. Journal of Quality Clinical Practice, 17(3), 123-129.

[25] Lethbridge-Cejku, M., Helmick, C.G. and Popovic, J. R. (2003) Hospitalizations for arthritis and other rheumatic conditions: Data from 1997. National Hospital Discharge Survey, 41(12), 1367-1373.

[26] Liu, B., Balkwill, A., Banks, E., Cooper, C., Green, J. and Beral, V. (2007) Relationship of height, weight and body mass index to the risk of hip and knee replacements in middle-aged women. Rheumatology, 46(5), 861-867.
[27] Greenfield, S., Apolone, G., McNeil, B.J. and Cleary, P.D. (1993) The importance of co-existent disease in the occurrence of postoperative complications and one-year recovery in patients undergoing total hip replacement. Medical Care, 31(2), 141-154.

[28] Gupta, S., Hawker, G.A., Laporte, A., Croxford, R. and Coyte, P.C. (2005) The economic burden of disabling hip and knee osteoarthritis (OA) from the perspective of individuals living with this condition. Rheumatology $(\mathrm{Ox}-$ ford), 44(12), 1531-1537.

[29] Theis, K.A., Helmick, C.G. and Hootman, J.M. (2007) Arthritis burden and impact are greater among U.S. women than men: Intervention opportunities. Journal of Womens Health, 16(4), 441-453. 